\title{
Clinical and Genetic Aspects of Juvenile Onset Pompe Disease
}

\author{
Johanna Holzwarth ${ }^{1}$ Nadja Minopoli ${ }^{1}$ Charlotte Pfrimmer ${ }^{1}$ Martin Smitka ${ }^{2}$ Sabine Borrel ${ }^{3}$ \\ Janbernd Kirschner $^{3}$ Nicole Muschol ${ }^{4}$ Hans Hartmann $^{5}$ Julia B. Hennermann ${ }^{6}$ Bernd A. Neubauer ${ }^{1}$ \\ Elke Hobbiebrunken ${ }^{7}$ Ralf A. Husain ${ }^{8}$ Andreas Hahn ${ }^{1}$
}

${ }^{1}$ Department of Child Neurology, Justus-Liebig University Gießen, Germany

${ }^{2}$ Children's hospital, Medical Faculty Carl Gustav Carus, Technische Universität Dresden, Dresden, Germany

3 Department of Neuropediatrics and Muscle Disorders, Medical Center-University of Freiburg, Freiburg, Germany

${ }^{4}$ Department of Pediatrics, University Medical Center HamburgEppendorf, Hamburg, Germany

${ }^{5}$ Hannover Medical School, Clinic for Pediatric Kidney, Liver and Metabolic Diseases, Hannover, Germany

Neuropediatrics 2022;53:39-45.

\author{
Address for correspondence Andreas Hahn, Abt. Kinderneurologie, \\ Sozialpädiatrie und Epileptologie des Zentrums Kinderheilkunde der \\ Justus-Liebig-Universität Gießen, Feulgenstr. 10-12, D-35392 Gießen, \\ Germany (e-mail: andreas.hahn@paediat.med.uni-giessen.de). \\ ${ }^{6}$ Villa Metabolica, Department of Pediatric and Adolescent Medicine, \\ University Medical Center Mainz, Mainz, Germany \\ 7 Department of Pediatrics and Adolescent Medicine, Division of \\ Pediatric Neurology, University Medical Center Göttingen, Georg \\ August University, Göttingen, Germany \\ ${ }^{8}$ Centre for Inborn Metabolic Disorders, Department of \\ Neuropediatrics, Jena University Hospital, Jena, Germany
}

\begin{abstract}
Keywords

- Pompe disease

- glucosidase

- phenotype

- genotype

Little is known about clinical symptomatology and genetics of juvenile onset Pompe disease (JOPD). The aims of this study were to analyze how these children are diagnosed, what clinical problems they have, and how phenotype is related to genotype. To accomplish this, we analyzed retrospectively data of 34 patients diagnosed after their first and before completion of their 18th birthday. Median age at diagnosis was 3.9 (range 1.1-17) years. Eight patients (23.5\%) developed initial symptoms in the first year, 12 (35\%) between 1 and 7 years, and $6(18 \%)$ thereafter. Eight (23.5\%) had no clinical symptoms at the time of diagnosis. Indications for diagnostics were a positive family history in three (9\%), hyperCKemia in eight (23.5\%), motor developmental delay in three (9\%), and muscle weakness and/or pain in 17 (50\%). Rare clinical signs were failure to thrive, recurrent diarrhea, and suspected hepatopathy with glycogen storage. Thirty-two different mutations were identified. Twenty-seven patients (79.5\%) carried the milder c.32-13T > G mutation, known to be associated with a broad range of phenotypes. Three out of eight patients manifesting within the first year of life showed generalized muscle weakness, hypertrophic cardiomyopathy, and had to be ventilated during the course of disease, thereby demonstrating clinical overlap with infantile onset Pompe disease.

These findings demonstrate that the phenotype of JOPD is broad and that the differential is not only restricted to neuromuscular disorders. Genotypic analysis was useful to delineate subjects with early onset JOPD from those with IOPD, but overall genotype-phenotype correlation was poor.
\end{abstract}

received

December 30, 2020

accepted after revision

July 27, 2021

published online

December 1, 2021 (c) 2021. Thieme. All rights reserved.

Georg Thieme Verlag KG,

Rüdigerstraße 14,

70469 Stuttgart, Germany
DOI https://doi.org/

10.1055/s-0041-1735250.

ISSN 0174-304X. 


\section{Introduction}

Pompe disease (glycogenosis type 2, acid maltase deficiency) is a rare metabolic myopathy caused by biallelic mutations of the acid $\alpha$-glucosidase (GAA) gene. ${ }^{1}$ This results in reduced activity of the lysosomal enzyme GAA with consecutive accumulation of glycogen preferentially in muscle and impaired autophagy. ${ }^{1-3}$ Pompe disease is usually divided into a rare infantile onset Pompe disease (IOPD) and a more frequent late onset Pompe disease type. ${ }^{1}$ The latter can be differentiated into adult Pompe disease (AOPD) and juvenile onset Pompe disease (JOPD). The incidence of IOPD is approximately $1: 140,000$ and that of all types together amounts to approximately 1:40,000 in Europe. ${ }^{1}$ Enzyme replacement therapy (ERT) is available since 2006 and has been shown to improve clinical symptoms in IOPD, JOPD, and AOPD. $^{4-6}$

IOPD is a multisystemic disease affecting several other tissues including the heart. Infants present with creatinine kinase (CK) elevation, severe hypertrophic cardiomyopathy (HCM), muscular hypotonia, and generalized muscle weakness usually during the first 6 months of life. ${ }^{1-4}$ This type is rapidly progressive and almost all subjects not treated with ERT die within the first year of life due to respiratory and/or cardiac failure. ${ }^{1-4}$ AOPD almost exclusively manifests as limb girdle muscular dystrophy often with early diaphragmatic involvement. ${ }^{1,6}$ While classic IOPD and AOPD represent relatively well characterized clinical entities, JOPD can manifest at any stage of motor development such as infancy, childhood, and adolescence. ${ }^{1,6,7}$ In addition, the implementation of new laboratory methods within the last years such as dried blood spot (DBS) testing and next-generation sequencing (NGS) has changed the diagnostic workup of subjects with limb girdle muscle weakness and/or CK-elevation, thereby facilitating identification of JOPD patients with minor or atypical clinical symptoms. ${ }^{2,3}$

Compared with IOPD and AOPD, little is known about the clinical symptomatology and the genetics of JOPD, and only one larger study analyzed this group of patients in more detail. ${ }^{7}$ Since detailed information is important for informed treatment decisions and correct counselling of families, the purpose of this study was to gain more insight in the phenotype and genotype of JOPD. A further aim was to analyze why and how these patients are diagnosed. To accomplish this, we analyzed retrospectively clinical and genetic data of a larger cohort of patients diagnosed with Pompe disease after the age of 1 and before the age of 18 years.

\section{Patients and Methods}

This study was approved by the ethical committee of the medical faculty of the Justus-Liebig-University Gießen, Germany. The parents of all patients and the patients themselves, if appropriate, gave formal consent for participation in this study. Inclusion criteria were a diagnosis of Pompe disease made after the first and before completion of the 18th birthday. A definite diagnosis was accepted if a patient showed a significantly reduced GAA activity in leukocytes or dried blood in conjunction with a pathogenic mutation on each allele of the GAA gene.

A questionnaire was designed containing items related to the age at onset of symptoms and diagnosis, mode of diagnostics, reasons to initiate diagnostics, symptoms present at diagnosis and at last follow-up, as well as results of genotyping. We contacted neuromuscular centers and institutions specialized in the care of patients with lysosomal storage disorders in Germany and asked for their consent to complete this questionnaire for all eligible patients. All patients included had a thorough neurologic examination by an experienced child neurologist or a specialist for metabolic disorders of childhood onset.

The patients' mutations were classified as very severe, potentially less severe, less severe, potentially mild, presumably nonpathogenic, and nonpathogenic according to information deposited in the Pompe variant database of the EMC in Rotterdam, The Netherlands (http://www.pompevariantdatabase.nl). This severity rating is based on molecular weight, amount, and residual enzymatic activity of the mutated protein when expressed in transfected COS-7 cells. ${ }^{8}$ The effects of variants not listed in this database were categorized as unknown.

Based on the age at onset of clinical symptoms, the cohort was divided into four groups: patients developing definite initial symptoms within the first year of life (infancy), from 1 to 7 years of age (childhood), after the age of 7 years (school age/adolescence), and patients without clinical symptoms at diagnosis. To analyze genotype-phenotype correlation in JOPD we compared the portion of very severe and potentially mild mutation between these groups. Statistical analysis was performed with SigmaPlot 11. Values are reported as median and range.

\section{Results}

Thirty-four patients from eight centers were included. Fifteen $(44 \%)$ were female. Median age at the diagnosis was 3.9 (range 1.1-17) years and that at last follow-up was 13.0 (range 2.1-22) years. Median age at manifestation of first clinical symptoms was 2.5 (range 0.5-17) years. Eight patients $(23.5 \%)$ showed initial clinical symptoms before the end of the first year of life (infancy), 12 (35\%) from the second to the seventh year (childhood), and six (18\%) subjects developed clinical signs thereafter (school age/adolescence). Eight individuals (23.5\%) had no clinical symptoms at the time of diagnosis (median age 5.9, range $1.5-17$ years) and four $(12 \%)$ continued to do so until their last follow-up (median duration 2.1, range 1-3.5 years). Twenty-seven patients (79.5\%) received ERT $(20 \mathrm{mg} / \mathrm{kg}$ rhGAA biweekly). In 24 subjects ERT was immediately started after the diagnosis had been established. Reasons to do so were overt muscle weakness in tandem with elevated CK in 15, motor developmental delay in conjunction with elevated $\mathrm{CK}$ in five, and muscle weakness together with HCM in three individuals. In a 17-year-old patient ERT was started because of hyperCKemia without clinical symptoms. 


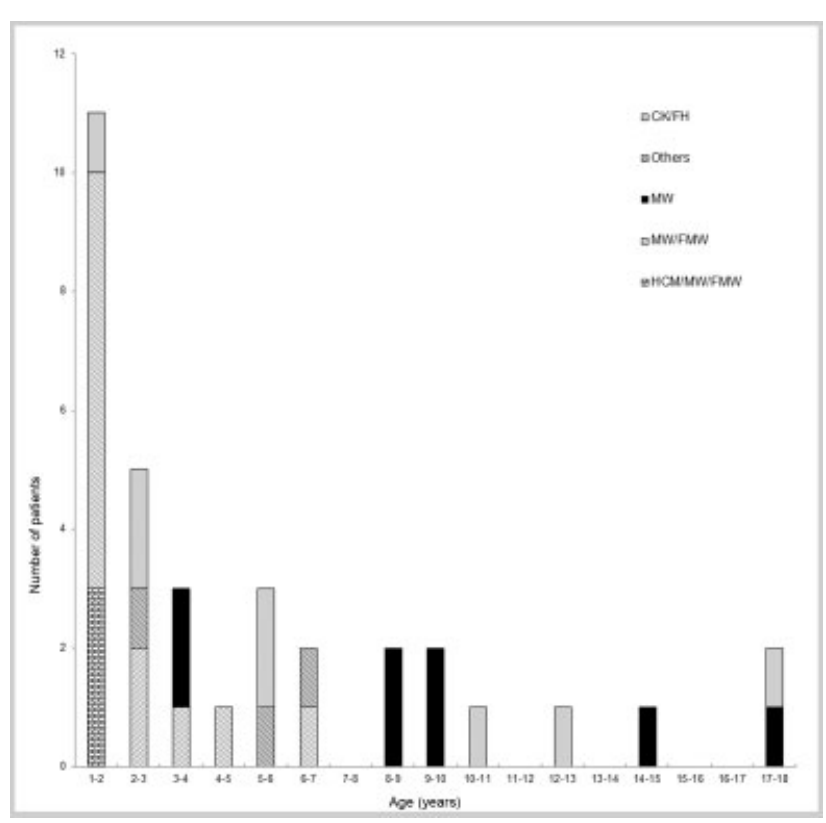

Fig. 1 Symptoms/reasons prompting diagnostics in relation to age at diagnosis of Pompe disease in 34 patients with juvenile onset. CK, CK elevation; FH, positive family history; FMW, facial muscle weakness; HCM, hypertrophic cardiomyopathy; MW, muscle weakness.

The seven patients who did not receive ERT until last followup included three patients with CK elevation without clinical symptoms, one patient for whom his parents refused ERT, and three Albanian siblings who went back to their home country and were lost in follow-up.

-Fig. 1 depicts the main symptoms/reasons prompting diagnostics in relation to age at diagnosis. While hyperCKemia or a family history positive for Pompe disease were reasons to initiate diagnostics at any age, the combination of HCM and facial/generalized muscle weakness was observed only in patients manifesting in infancy. Limb girdle muscle together with facial weakness was frequent in patients developing first symptoms during childhood, whereas facial weakness was rarer in those manifesting later. Indications for diagnostics were a positive family history in three (9\%), motor developmental delay in three (9\%), muscle weakness in 17 (50\%), and elevated CK-values in eight patients (23.5\%). In the latter group, hyperCKemia was noticed when taking blood because of pneumonia $(n=1)$, upper respiratory tract infection $(n=1)$, gastroenteritis $(n=2)$, fatigue resolving without specific treatment $(n=1)$, and surgery $(n=3)$. However, careful neurologic examination at the time of referral disclosed muscular hypotonia and mild muscle weakness in two of them. All patients had elevated CK values at diagnosis ranging from 229 to $8.390 \mathrm{U} / \mathrm{L}$ (median value $899 \mathrm{U} / \mathrm{L}$; normal $<200 \mathrm{U} / \mathrm{L}$ ), and 11 (32\%) complained about muscle pain.

In 17 patients (50\%) JOPD was diagnosed by GAA activity determination in leukocytes and in 14 (41\%) by DBS analysis. Three patients (9\%) were diagnosed by NGS applied to elucidate the reason for elevated $\mathrm{CK}$ values and/or limb girdle muscle weakness.
Three patients manifesting in the first year of life had mild HCM (left ventricular mass index less than one standard deviation above the upper limit), four developed severe scoliosis prompting spinal surgery, and in three ventilatory failure necessitated assisted ventilation during follow-up. In children manifesting during childhood, failure to thrive, chronic diarrhea, and suspected hepatopathy with glycogen storage was the initial sign prompting further diagnostics in one patient each. All three also had minimal CK-elevations (229-314 U/L) and displayed mild proximal muscle weakness when a careful neurologic examination was performed. One 8-year-old boy with onset of clinical symptoms after the age of 7 had a rigid spine.

Among the eight patients without clinical symptoms at the time of diagnosis, diagnostics were performed because of a positive family history in two and due to increased CK values in six. The four patients who remained symptom-free for at least 1 to 3.5 years were diagnosed at age 5 (two brothers), 12, and 17 years with CK values ranging from 260 to $750 \mathrm{U} / \mathrm{L}$. The three younger patients were not treated with ERT. The four patients who developed clinical symptoms were diagnosed at the age $1.5,2,2.5$, and 10.5 years, respectively. Their CK values varied from 295 to $760 \mathrm{U} / \mathrm{L}$. Limb girdle muscle weakness became obvious $0.5,1$, and 1.5 years after establishing the diagnosis despite immediate start of ERT in the three younger patients. In the oldest subject, difficulties in climbing were first documented 2 years after diagnosis, but the parents refused ERT until last follow-up.

All 34 patients (100\%) were alive at last follow-up. Motor function was not assessed in a standardized manner, but analysis of motor milestones and respiratory status revealed that all patients achieved sitting without support, and that 28 (82\%) learned to walk. Besides the three subjects remaining symptom-free during the observation period, four patients asymptomatic at diagnosis developed clinical symptoms (12\%), another five lost ambulation (15\%), and another five became ventilator-dependent (15\%). Overall, Pompe disease was progressive in 14 patients (41\%), and in 10 (30\%) progression occurred despite ERT.

Thirty-two different genetic GAA variants were detected (-Table 1). In one patient (number 28, -Table 1) with a distinctly reduced GAA activity in leukocytes, the sequence variant on one allele turned out not to result in an amino acid exchange. Five mutations were novel (three single patients, one pair of twins, and one set of triplets). Twenty-seven patients (79.5\%) carried the mild c.32-13T $>$ G mutation on one allele. Overall, 29 patients (85\%) showed a mutation listed as potentially mild in the Pompe variant database on one allele. In 14, this was combined with a very severe, in six with a less or potentially less severe, and in two with another potentially mild mutation on the second allele. Four patients had a combination of two less or potentially less severe mutations, while one patient each had a combination of two mutations with unknown effects, or a potentially mild mutation on one and a novel mutation on the other allele. CRIM status was positive in seven out of eight patients manifesting in the first year of life and was unknown in 


\begin{tabular}{|c|c|c|c|c|c|c|c|c|c|c|c|c|c|c|c|c|c|c|c|c|c|c|c|}
\hline & 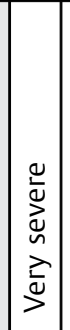 & $\begin{array}{l}\frac{5}{3} \\
\frac{5}{5} \\
\frac{5}{5}\end{array}$ & 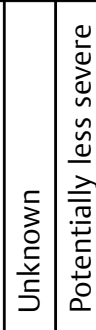 & 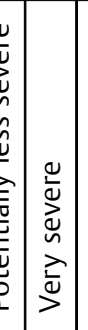 & $\ddot{\bar{z}}$ & 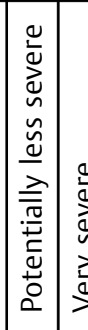 & 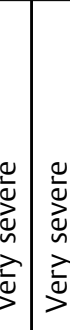 & 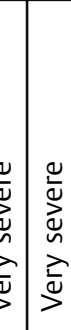 & 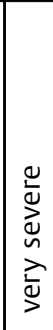 & 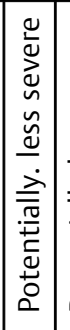 & 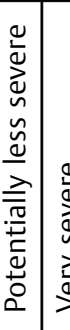 & 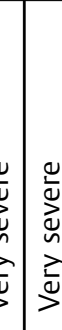 & 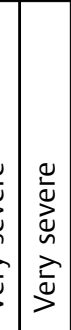 & 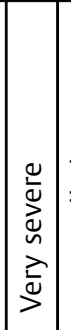 & 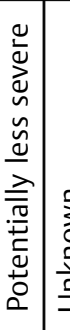 & 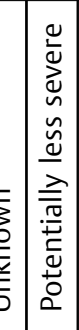 & & 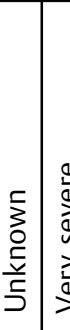 & 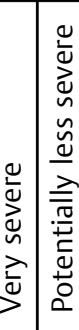 & 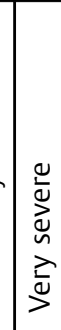 & 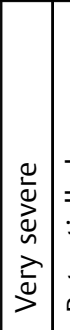 & 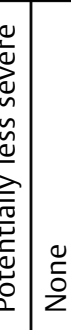 & \\
\hline & 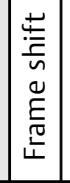 & 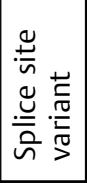 & 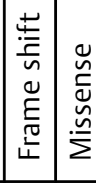 & 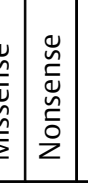 & 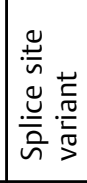 & 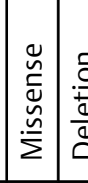 & & 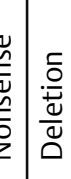 & & \begin{tabular}{|l|} 
\\
$\grave{\omega}$ \\
$\bar{\omega}$ \\
$\stackrel{\tilde{\nu}}{\Sigma}$ \\
\end{tabular} & 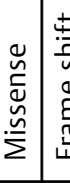 & & 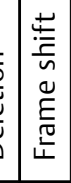 & 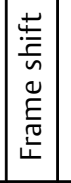 & 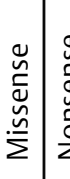 & 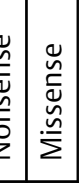 & 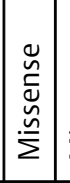 & 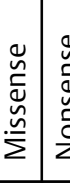 & 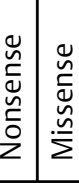 & 莺 & $\begin{array}{l}\text { 总 } \\
\text { 离 }\end{array}$ & & \\
\hline
\end{tabular}

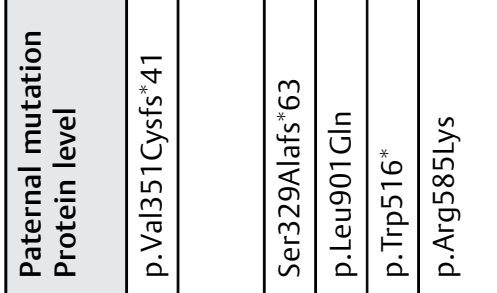

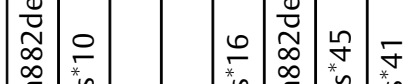

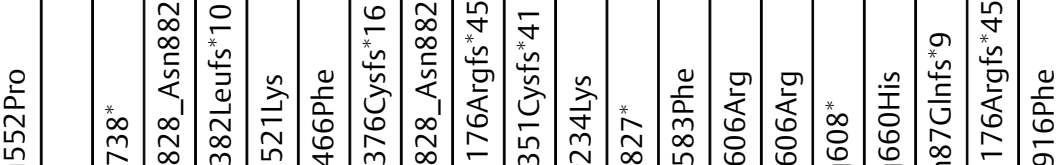

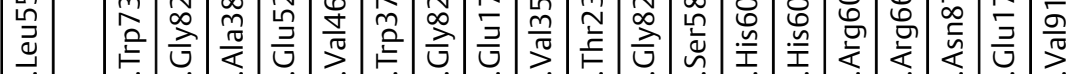

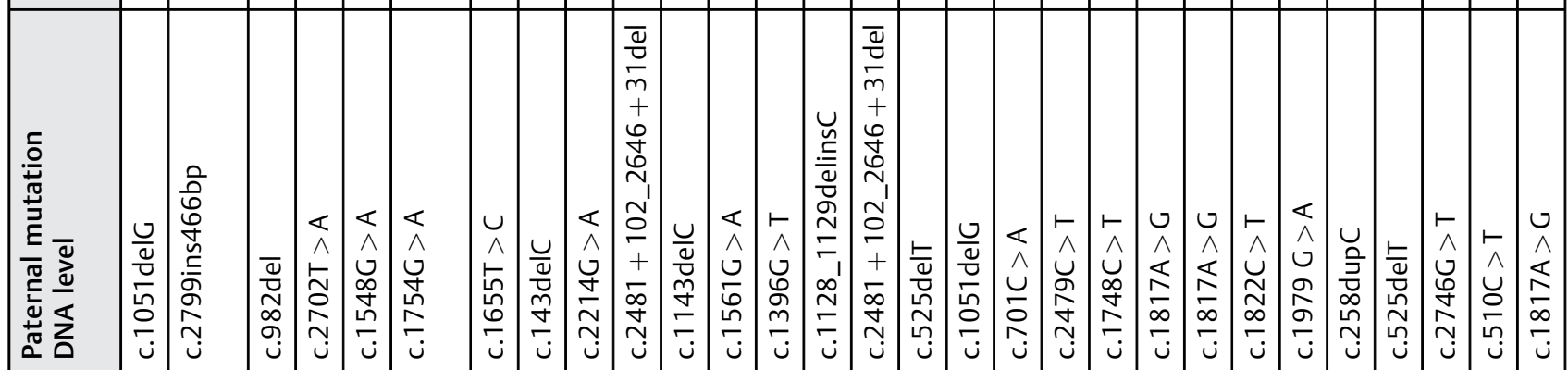

\begin{tabular}{|c|c|c|c|c|c|c|c|c|c|c|c|c|c|c|c|c|c|c|c|c|c|c|c|}
\hline & 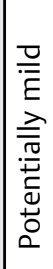 & \begin{tabular}{|l}
$\mid \frac{5}{3}$ \\
o. \\
$\frac{5}{5}$ \\
$\frac{5}{5}$
\end{tabular} & 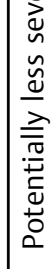 & 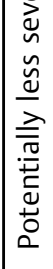 & 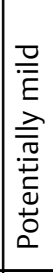 & 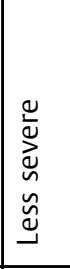 & 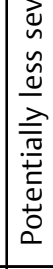 & 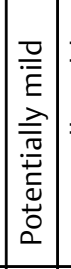 & 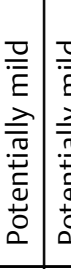 & 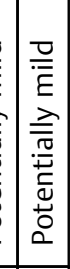 & 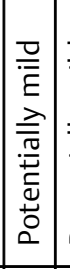 & 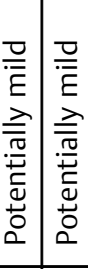 & 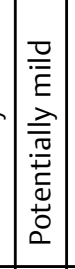 & 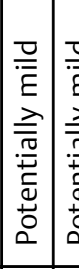 & 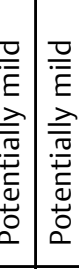 & 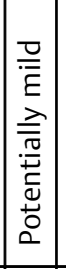 & 节 & 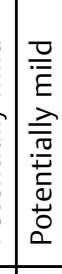 & 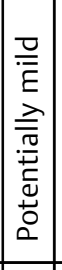 & 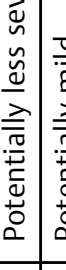 & $\frac{\partial}{\bar{\Sigma}}$ & 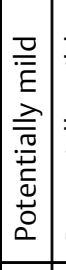 & 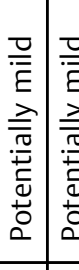 \\
\hline & 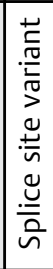 & 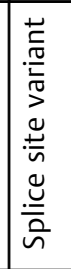 & 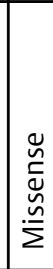 & 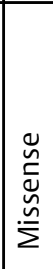 & 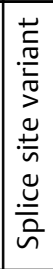 & 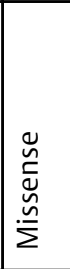 & 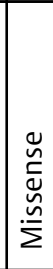 & 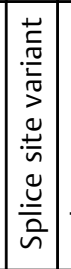 & 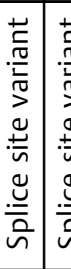 & 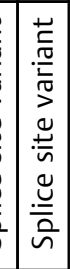 & 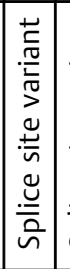 & 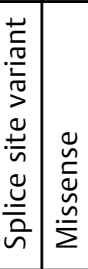 & 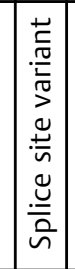 & 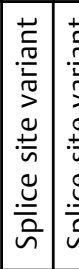 & 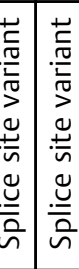 & 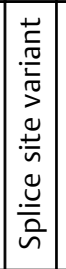 & $\mid$ & 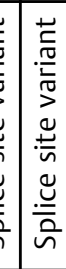 & 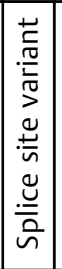 & 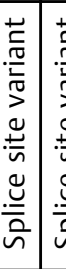 & 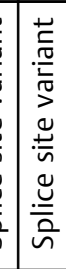 & 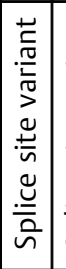 & 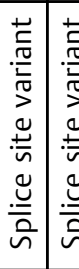 \\
\hline 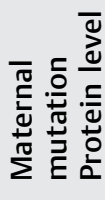 & & & 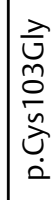 & 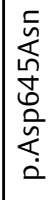 & & 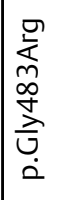 & 15 & & & & & & & & & & 高 & & & & & & \\
\hline 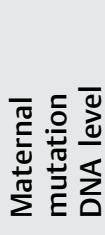 & 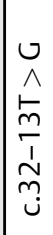 & 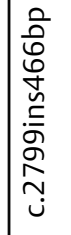 & $\begin{array}{l}u \\
\hat{N} \\
\hat{今} \\
\tilde{O}\end{array}$ & $\mid \begin{array}{l}\hat{\wedge} \\
\hat{N} \\
\tilde{n} \\
\tilde{n}\end{array}$ & $\mid \begin{array}{l}u \\
\hat{N} \\
\stackrel{5}{n} \\
1 \\
\tilde{N} \\
ن\end{array}$ & 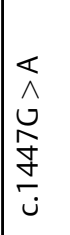 & 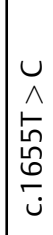 & 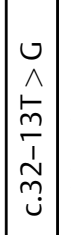 & 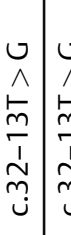 & 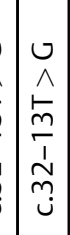 & 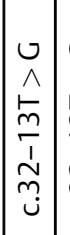 & 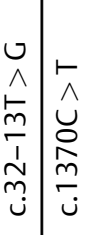 & 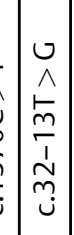 & 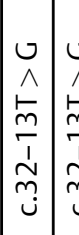 & 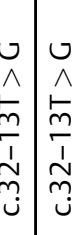 & 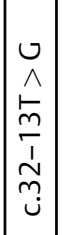 & 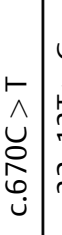 & $\stackrel{\cup}{\stackrel{1}{\prime}}$ & $\mid \begin{array}{l}0 \\
\hat{n} \\
\frac{\hat{m}}{1} \\
\end{array}$ & & 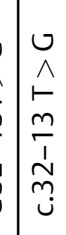 & $\left|\begin{array}{l}\hat{\lambda} \\
\hat{\underline{m}}\end{array}\right|$ & 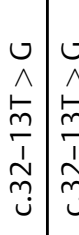 \\
\hline & & & & & & & & & $a$ & & $\simeq$ & $\stackrel{m}{2}$ & & & & & $\stackrel{\sim}{\sim}$ & & & & & & \\
\hline
\end{tabular}




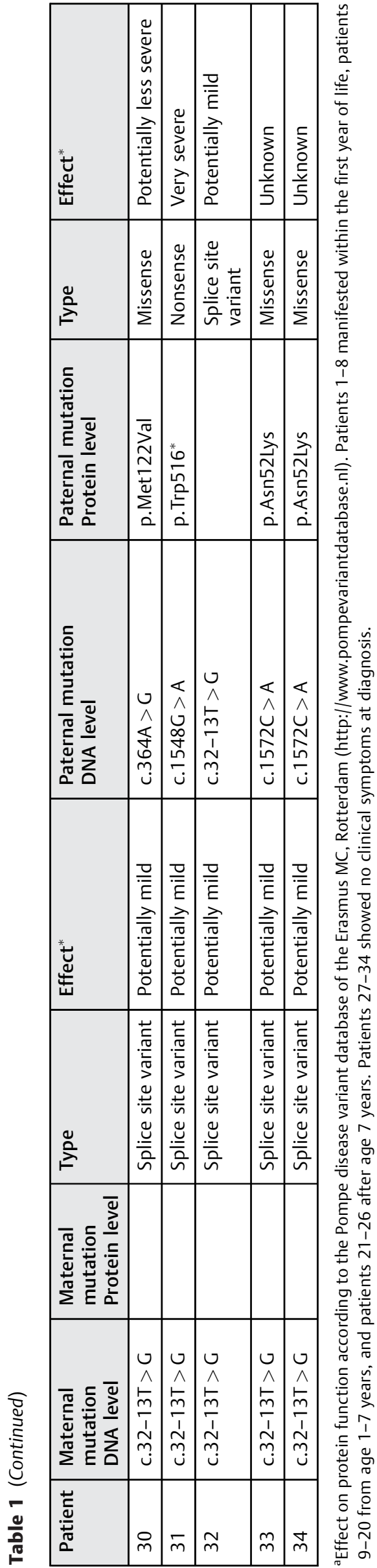

the remaining patient harboring a homozygous intronic splice site variant. No patient received immunomodulation. The number of patients with very severe and potentially mild GAA mutations in relation to age at the onset or presence of clinical symptoms at diagnosis is shown in - Table 2.

\section{Discussion}

In this study we analyzed retrospectively phenotypic and genotypic findings of 34 German Pompe patients diagnosed after their first and before their 18th birthday. Our findings confirm that JOPD is a very heterogeneous condition with a broad phenotype and a progressive course in many patients, which makes general comments and conclusions difficult. ${ }^{7}$

Mild HCM and ventilatory failure during follow-up in some patients manifesting during infancy demonstrate overlap with IOPD. As done in this study, such patients can be classified as JOPD since all were diagnosed after their first birthday. Alternatively they can be catogrized as suffering from nonclassic IOPD since first symptoms occurred during the first year of life. The latter term was coined in the pre-ERT era by Slonim and colleagues who delineated a group of patients with onset of symptoms in the first year of life, mild HCM, and an overall better prognosis from infants with classic IOPD. ${ }^{9}$ The relatively large number of individuals showing symptoms already in the first year of life is in line with observations made by van Capelle et al reporting 31 subjects with JOPD ${ }^{7}$ and suggests that symptom onset before the first birthday is not infrequent in JOPD. Proximal myopathy together with or without facial weakness, moderate CKelevation, and motor problems were typical features of JOPD manifesting later. However, unusual reasons to perform diagnostics (e.g., suspected liver disease, chronic diarrhea, and failure to thrive) and atypical symptoms (e.g., rigid spine) indicate that the differential of JOPD is broad and not only restricted to neuromuscular disorders. Less than one-fifth of our patients developed clinical symptoms after age 7 , reflecting that Pompe disease is only rarely diagnosed in school children and adolescents.

Notably, almost one-quarter of patients was asymptomatic at diagnosis. Such a relatively high proportion has also been reported by Capelle et $\mathrm{al}^{7}{ }^{7}$ and probably reflects changed diagnostic practices in patients with CK-elevation. Today, enzyme activity determination from dried blood by fluorometry or tandem mass spectrometry is a cost-effective and simple method that is now widely used in a variety of metabolic diseases. ${ }^{10}$ A study screening of 3,076 adults with CK-elevation and/or limb girdle weakness by DBS for reduced GAA activity identified 74 patients (2.4\%) with Pompe disease, ${ }^{11}$ while a similar investigation in 37 children yielded a detection rate of $4.5 \%{ }^{12}$ Based on such studies, GAA activity measurement in DBS is now frequently applied to rule out Pompe disease as a potentially treatable neuromuscular disorder. ${ }^{3}$ Similarly, NGS that allows analyzing a greater number of genes in parallel for defects causing a similar unspecific phenotype is increasingly performed to elucidate the exact cause of limb girdle muscle weakness. ${ }^{13}$ All patients in our cohort had hyperCKemia, underscoring that 
Table 2 Genotype data in relation to age at onset or presence of clinical symptoms at diagnosis

\begin{tabular}{|l|l|l|l|l|}
\hline & \multicolumn{4}{|l|}{ Age at onset of symptoms/no symptoms at diagnosis } \\
\hline & $\begin{array}{l}<\mathbf{1} y \\
\text { (infancy) } \\
n=8\end{array}$ & $\begin{array}{l}1-7 \mathrm{y} \\
\text { (childhood) } \\
n=12\end{array}$ & $\begin{array}{l}\text { 7-18 y } \\
\text { (school age/adolescence) } \\
n=6\end{array}$ & $\begin{array}{l}\text { No symptoms } \\
n=8\end{array}$ \\
\hline Very severe & $3(37.5 \%)$ & $7(58 \%)$ & $3(50 \%)$ & $0(0 \%)$ \\
\hline Potentially mild & $3(37.5 \%)$ & $11(92 \%)$ & $4(67 \%)$ & $8(100 \%)$ \\
\hline
\end{tabular}

the diagnosis of Pompe disease is unlikely in children without hyperCKemia. 5,7

In IOPD, there is no robust evidence of which ERT dosing schedule is most effective, ${ }^{14}$ and there is an increasing amount of arguments suggesting that higher doses of recombinant GAA up to $40 \mathrm{mg} / \mathrm{kg}$ body weight every week are more effective than the recommended $20 \mathrm{mg} / \mathrm{kg}$ body weight every other week. ${ }^{15-17}$ The severe clinical symptoms and the progressive course in some of our patients manifesting in the first year of life raise the question whether such a high dose therapy could also be an option for this subgroup of JOPD patients.

Criteria when to start ERT have been defined for adults with Pompe disease but not yet for patients with JOPD. ${ }^{18}$ However, it seems to be accepted that children should be treated when they are symptomatic, i.e., when they have skeletal muscle weakness or respiratory muscle involvement as observed using clinical assessments. ${ }^{3}$ In contrast, increased CK per se is not regarded as an indication for ERT. ${ }^{18}$

In principle, the effects of the underlying genetic defect could be used as an additional criterion whether to treat or not. In Pompe disease it is assumed that age at diagnosis reflects disease severity and corresponds to residual GAA enzyme activity, which in turn depends on the underlying genetic defect. ${ }^{1}$ However, the initial symptom prompting diagnostics (e.g., CK-elevation) can lead to an early diagnosis in a preclinical state, whereas failure to recognize that a specific complaint (e.g., muscle pain) represents a symptom of disease can substantially protract the diagnosis. Additionally, modifying variables such as genetic, epigenetic, and environmental factors can influence manifestation of the disease $e^{19-21}$

In this study, comparing the rate of very severe mutations between the different age groups (infancy, childhood, school age/adolescence) revealed that these numbers were similar. Although no patient without clinical symptoms at diagnosis carried such a mutation this suggests that the finding of one very severe mutation in a patient with JOPD is not per se associated with an early onset of symptoms or a more severe course of disease. Similar to what is seen in AOPD and in marked contrast to what is found in IOPD, ${ }^{20}$ approximately $85 \%$ of our patients carried a potentially mild mutation on at least one allele, and in almost four-fifths this was the intronic c.32$13 \mathrm{~T}>\mathrm{C}$ mutation. While this genetic variant results in some residual enzyme activity probably preventing manifestation as IOPD, ${ }^{22}$ it is known to be associated with a broad range of phenotypes, ${ }^{21-23}$ rendering prediction for the further course of disease difficult. Concluding from genotype to phenotype in our cohort was also impeded by the detection of five mutations not yet characterized at the biochemical level. These results are in good congruence with the findings of Capelle et $\mathrm{al}^{7}$ and with the study of Reuser and colleagues who analyzed GAA variants and phenotype among more than 1,000 patients with Pompe disease included in the Pompe registry. ${ }^{24}$

Our study has some shortcomings. Although this is the largest JOPD cohort analyzed so far, the number of patients is still small. In addition, this was a retrospective study collecting data from different centers. Moreover, we included only patients diagnosed until completion of their 18th birthday. An analysis of the Pompe registry data revealed that the median time gap between onset of symptoms and diagnosis in individuals manifesting during adolescence ( $>12$ years) was 12.6 years. $^{25}$ Therefore, it has to be assumed that a substantial number of patients with first symptoms in adolescence were missed by our approach.

In conclusion, this study showed that genotyping helps to distinguish JOPD from IOPD, but that overall genotype-phenotype correlation in JOPD is poor, and that it does not add much to predicting disease severity or making treatment decisions. The decision when to start ERT in an individual JOPD patient without overt clinical symptoms cannot be based on a single parameter alone but should rather take into consideration different aspects such as age, course of disease in siblings if known, CK-level, other paraclinical parameters (e.g., muscle biopsy and MRI), and genetic findings.

\section{Conflict of Interest}

None declared.

\section{References}

1 van der Ploeg AT, Reuser AJ. Pompe's disease. Lancet 2008;372 (9646):1342-1353

2 Hahn A, Schänzer A. Long-term outcome and unmet needs in infantile-onset Pompe disease. Ann Transl Med 2019;7(13):283

3 Hahn A, Hennermann JB, Huemer M, et al. Diagnosis and care of infants and children with Pompe disease. Klin Padiatr 2020;232 (02):55-61

4 Kishnani PS, Corzo D, Nicolino M, et al. Recombinant human acid $[\alpha]$-glucosidase: major clinical benefits in infantile-onset Pompe disease. Neurology 2007;68(02):99-109

5 van der Meijden JC, Kruijshaar ME, Harlaar L, Rizopoulos D, van der Beek NAME, van der Ploeg AT. Long-term follow-up of 17 patients with childhood Pompe disease treated with enzyme replacement therapy. J Inherit Metab Dis 2018;41(06):1205-1214

6 van der Ploeg AT, Clemens PR, Corzo D, et al. A randomized study of alglucosidase alfa in late-onset Pompe's disease. N Engl J Med 2010;362(15):1396-1406

7 van Capelle CI, van der Meijden JC, van den Hout JMP, et al. Childhood Pompe disease: clinical spectrum and genotype in 31 patients. Orphanet J Rare Dis 2016;11(01):65 
8 Kroos M, Pomponio RJ, van Vliet L, et al; GAA Database Consortium. Update of the Pompe disease mutation database with 107 sequence variants and a format for severity rating. Hum Mutat 2008;29(06):E13-E26

9 Slonim AE, Bulone L, Ritz S, Goldberg T, Chen A, Martiniuk F. Identification of two subtypes of infantile acid maltase deficiency. J Pediatr 2000;137(02):283-285

10 Hong X, Sadilek M, Gelb MH. A highly multiplexed biochemical assay for analytes in dried blood spots: application to newborn screening and diagnosis of lysosomal storage disorders and other inborn errors of metabolism. Genet Med 2020;22(07):1262-1268

11 Lukacs Z, Nieves Cobos P, Wenninger S, et al. Prevalence of Pompe disease in 3,076 patients with hyperCKemia and limb-girdle muscular weakness. Neurology 2016;87(03):295-298

12 Ünver O, Hacıfazlıoğlu NE, Karatoprak E, et al. The frequency of late-onset Pompe disease in pediatric patients with limb-girdle muscle weakness and nonspecific hyperCKemia: a multicenter study. Neuromuscul Disord 2016;26(11):796-800

13 Savarese M, Torella A, Musumeci O, et al. Targeted gene panel screening is an effective tool to identify undiagnosed late onset Pompe disease. Neuromuscul Disord 2018;28(07):586-591

14 Chen M, Zhang L, Quan S. Enzyme replacement therapy for infantile-onset Pompe disease. Cochrane Database Syst Rev 2017;11:CD011539

15 van Gelder CM, Poelman E, Plug I, et al. Effects of a higher dose of alglucosidase alfa on ventilator-free survival and motor outcome in classic infantile Pompe disease: an open-label single-center study. J Inherit Metab Dis 2016;39(03):383-390

16 Case LE, Bjartmar C, Morgan C, et al. Safety and efficacy of alternative alglucosidase alfa regimens in Pompe disease. Neuromuscul Disord 2015;25(04):321-332
17 Desai AK, Walters CK, Cope HL, Kazi ZB, DeArmey SM, Kishnani PS. Enzyme replacement therapy with alglucosidase alfa in Pompe disease: clinical experience with rate escalation. Mol Genet Metab 2018;123(02):92-96

18 van der Ploeg AT, Kruijshaar ME, Toscano A, et al; European Pompe Consortium. European consensus for starting and stopping enzyme replacement therapy in adult patients with Pompe disease: a 10-year experience. Eur J Neurol 2017;24(06):768-e31

19 Bergsma AJ, In 't Groen SLM, van den Dorpel JJA, et al. A genetic modifier of symptom onset in Pompe disease. EBioMedicine 2019;43:553-556

20 Kroos MA, Pomponio RJ, Hagemans ML, et al. Broad spectrum of Pompe disease in patients with the same c.-32-13T- $>$ G haplotype. Neurology 2007;68(02):110-115

21 Wens SC, van Gelder CM, Kruijshaar ME, et al. Phenotypical variation within 22 families with Pompe disease. Orphanet J Rare Dis 2013;8:182

22 Niño MY, In 't Groen SLM, Bergsma AJ, et al. Extension of the Pompe mutation database by linking disease-associated variants to clinical severity. Hum Mutat 2019;40:1954-1967

23 Dardis A, Zanin I, Zampieri S, et al. Functional characterization of the common c.-32-13T>G mutation of GAA gene: identification of potential therapeutic agents. Nucleic Acids Res 2014;42(02):1291-1302

24 Reuser AJJ, van der Ploeg AT, Chien YH, et al; On Behalf Of The Pompe Registry Sites. GAA variants and phenotypes among 1,079 patients with Pompe disease: data from the Pompe Registry. Hum Mutat 2019;40(11):2146-2164

25 Kishnani PS, Amartino HM, Lindberg C, Miller TM, Wilson A, Keutzer JPompe Registry Boards of Advisors. Timing of diagnosis of patients with Pompe disease: data from the Pompe registry. Am J Med Genet A 2013;161A(10):2431-2443 